Laser Chem., 2002, Vol. 20(1), pp. 1-21

Taylor \& Francis

Taylor \& Francis Group

\title{
LASER AND MATERIAL PARAMETER DEPENDENCE OF THE CHEMICAL MODIFICATIONS IN THE UV LASER PROCESSING OF MODEL POLYMERIC SOLIDS
}

\author{
E. ANDREOU, A. ATHANASSIOU, D. FRAGOULI, \\ D. ANGLOS* and S. GEORGIOU* \\ Institute of Electronic Structure and Laser, Foundation for Research and \\ Technology-Hellas, P.O. Box 1527, 71110 Heraklion, Crete, Greece
}

(Received 21 May 2001)

\begin{abstract}
Chemical modifications are expected to be the major type of side-effect in the UV laser processing of molecular substrates. For their systematic characterization, studies on polymeric systems consisting of poly(methyl methacrylate) and polystyrene films doped with aromatic dopants exemplifying different degrees of photoreactivity are undertaken. In particular, the dependence of the nature and extent of the modifications on chromophore properties and laser parameters (laser fluence, wavelength, and number of pulses) is examined. The substrate absorptivity and the number of employed laser pulses turn out to be the critical parameters in determining the quantity and nature of photoproducts that remain in the substrate. The implications of these results for the optimisation of laser processing of molecular/organic solids are discussed. It is suggested that the importance of employing relatively strongly absorbed wavelengths in laser processing may relate, besides the efficient etching and good surface morphology, to the minimization of the chemical modifications. In contrast, irradiation with successive laser pulses is indicated to be highly disadvantageous for the chemical integrity of the substrate. In all, the study of such model systems appears to be most appropriate for establishing criteria for the systematic optimisation of laser processing techniques of molecular substrates.
\end{abstract}

Keywords: UV ablation; Molecular substrates; Polymers; Photochemistry; Restoration of artworks

\footnotetext{
* Corresponding authors. E-mail: anglos@iesl.forth.gr
} 


\section{INTRODUCTION}

Due to its several advantages over traditional methods of material removal, UV ablation has found extensive use for the processing of polymeric substrates. In parallel, new applications are continuously being introduced. For instance, ablation is used for the patterning of polymers [1-3], for the removal of microparticles in microelectronic industry [4], for polymer deposition [5] as well as for dry image production via laser ablation transfer [6]. The particular advantages of UV ablation for demanding processing purposes are clearly illustrated by its successful use for the restoration of the highly sensitive painted artworks [7, 8]. By now, there are several examples in which laser processing has enabled the restoration of painted artworks that would not be amenable to treatment by traditional/ conventional techniques. Closely related to these applications and further demonstrating the potential of UV ablation is its use for the precise excision of tissue in medicine, as for example in photorefractive keratectomy [9-11].

For the development of these applications, extensive work has been performed for establishing optimal processing parameters. In most cases, the optimisation has been achieved empirically, largely on a trial-and-error basis. This is due to the ill-understood nature of the effects of UV ablation. In particular, the processes that underline UV ablation appear to differ significantly from those taking place at low laser intensities, so that the principles of conventional photochemistry are insufficient in guiding the efforts. For instance, in many cases, the processing of chemically similar systems calls for considerable changes in the employed parameters and/ or procedures. Clearly, as the applications of UV ablation become more sophisticated and applied to more "sensitive" substrates, there is strong need for the specification of well-defined criteria for guiding the systematic selection of processing parameters. To this end, a detailed examination of the effects of the ablation process and of their dependence on processing parameters is undertaken.

A major advantage of employing UV light for the processing of molecular substrates is considered to be the limited thermal "load" to the substrate. On the other hand, the use of high intensity UV laser pulses raises serious concerns in relationship with the photochemical modifications that may be induced to the substrate. In most applications, the processed molecular substrates include a wide variety of chromophores. Upon photoexcitation, several of the included chromophores dissociate into highly reactive fragments, 
which can subsequently react with nearby units and/or form oxidation products. In parallel, the relatively high temperatures attained $\left(\geq 800^{\circ} \mathrm{C}\right)[12$, 13] may result in thermal dissociation of the weaker bonds and further production of radicals. Finally, the high amplitude stress waves (of the order of GPa) developed may further enhance reactivity [14-16]. It is also important to note that as all these factors operate in parallel, there can be a "positive feedback" between them [17] and the finally produced chemical effects may far exceed a simple "summation" of their contributions. For these reasons, UV ablation might be expected to result in extensive photochemical modifications, with detrimental effects on the integrity of the substrates.

It is clear that a thorough characterization of the chemical effects and of their dependence on laser-processing parameters is difficult to achieve in the chemically and structurally complex substrates (i.e., painted artworks, tissue, etc.) encountered in the above applications. In an attempt to overcome this problem, we have been performing studies on model polymeric systems, namely polymer films doped with simple chromophores. Poly(methyl methacrylate), PMMA and polystyrene, PS have been used as host polymers. Various organic chromophores have been employed, with the work focussed on simple aromatic compounds, namely naphthalene, phenanthrene and their halo-derivatives (bromo- and iodo-derivatives). These doped polymer systems constitute a good model for a number of systems encountered in the various applications of UV ablation. For instance, they present close analogies to the painting medium in artworks, where pigments are dispersed within an organic polymerised medium [7], or to biological tissues that include a variety of proteins and other biological chromophores within a collagen stroma [9]. On the other hand, as compared with these realistic systems, the chosen systems offer the advantage of the chemical simplicity of the dopants, thereby enabling the systematic monitoring of their modifications. Furthermore, the well-known photochemistry of the dopants provides a basis for the elucidation of the factors responsible for the induced photochemical effects.

Three laser parameters are usually of consideration in the various processing applications, namely wavelength, fluence and number of pulses applied to a given area $[1,2]$. Here, we assess the importance of all three parameters in determining the nature and extent of the induced modifications to the dopants in the substrate. UV ablation with nanosecond pulses is shown to result in considerable modifications, in particular formation of recombination (i.e., products through reactions of two dopant molecules 
or radicals) and polycyclic fused products. No particular dependence of the nature, at least of the emitting products probed here, on wavelength is observed. On the other hand, different types of $\mathrm{F}_{\text {LASER }}$-dependence of the photoproduct amount remaining in the substrate are found for the particular systems at different wavelengths. In all cases, the extent of chemical modifications deviates much from that observed at low fluences, but in different ways at the various wavelengths. It is shown that absorptivity of the substrate at the irradiation wavelength is the major determinant for the type of dependence observed (i.e., chemical composition of the polymer does not appear, in first approximation, to be as significant). Successive laser pulses result in further photolysis and accumulation of photoproducts, as well as in secondary, ill-defined photoproducts. The results are correlated with the procedures adapted in the various laser processing schemes of molecular solids, taking as an example the laser restoration of painted artworks. In particular, the results suggest that one of the reasons for the importance of employing strongly absorbed wavelengths, besides the efficient etching and the good surface morphology, is the minimization of photochemical modifications in the substrate.

\section{EXPERIMENTAL}

Highly purified poly(methyl methacrylate) $\left(M_{w} \sim 120,000\right)$ and polystyrene $\left(M_{w} \sim 280,000\right)$ are doped with the aromatics naphthalene $(\mathrm{NapH})$ and phenanthrene $(\mathrm{PhenH})$ and their iodo-derivatives 1-iodonaphthalene (NapI) and 9-iodophenanthrene (PhenI) (Aldrich). For the preparation of the films, appropriate amounts of the purified polymer and of the dopant are dissolved into dichloromethane $\left(\mathrm{CH}_{2} \mathrm{Cl}_{2}\right)$. Subsequently the solutions are cast on quartz substrates. The dopant concentration varies from $0.1 \%$ to $4.0 \%$ by weight and the typical film thickness is in the range of 10 $80 \mu \mathrm{m}$, as measured by a profilemeter.

For the characterization and quantification of the induced chemical modifications, laser-induced fluorescence is employed to interrogate the irradiated polymer samples. This examination takes advantage of the fact that the products formed by the photolysis of the NapI and PhenI precursors are generally highly fluorescing [18]. Similarly, polystyrene and the products of the PMMA degradation are fluorescing and can conveniently be monitored via laser-induced fluorescence. Accordingly the experiment is of the pump- 
probe type, where the pump pulse effects ablation and the probe beam induces the emission of the photoproducts that remain in the substrate.

"Pumping" (i.e. photoproduct formation) is effected with a nanosecond $\mathrm{KrF}$ excimer laser (Lambda-Physik Compex 110) operating at 248 and $193 \mathrm{~nm}$, respectively or a XeCl excimer laser (Lambda-Physik LPX 300) operating at $308 \mathrm{~nm}$. The laser beam is focused perpendicularly onto the sample in a rectangular spot of $6-10 \mathrm{~mm}^{2}$, using a quartz spherical lens $(f=+500 \mathrm{~mm})$. Irradiation of the samples is performed in ambient atmosphere as this is the case in most UV laser processing applications. After irradiation of the doped films with a given number of pump pulses, photoproduct fluorescence is induced by excitation with $248 \mathrm{~nm}$ laser pulses of very low fluence $\left(\mathrm{F}_{\mathrm{LASER}} \leq 3 \mathrm{~mJ} \mathrm{~cm} \mathrm{~cm}^{-2}\right.$ ). Thus, photoproduct formation by the probe beam itself is negligible and all recorded fluorescence can be assumed to derive exclusively from photoproducts formed by the previous "pump" pulse. In the experiments in which ablation is also performed at $248 \mathrm{~nm}$, probing is effected with exactly the same beam configuration (except for the decreased fluence of the probe beam) and thus the ablated and probed areas are identical. In this case, the main source of error in the fluorescence intensity measurements/comparison derives mainly from variations in the thickness (and thus amount of photoproduct formed) across a given film (usually of $\sim 20 \%$ ). For the experiments in which the ablation is performed at $193 \mathrm{~nm}$ or $308 \mathrm{~nm}$, probed and ablated areas are nearly matched. However, differences can not be excluded and thus the quantitative comparison between different wavelengths is subject to this additional source of error, though this is expected to be small.

A relatively long delay (of the order of several seconds) between the pump and probe pulses is employed for ensuring nearly quantitative reaction of the photoinduced radicals and formation of stable photoproducts. Indeed, the photoproduct fluorescence intensity remains constant for longer delay times.

The emission induced by the probe beam is collected by an optical fibre oriented nearly perpendicularly to the sample and at a distance of 1 to $2 \mathrm{~cm}$ away from its surface. An $x-y$ micrometer ensures accurate positioning of the optical fibre relatively to the irradiated spot. The light from the fibre is spectrally analysed in a $0.20 \mathrm{~m}$ grating spectrograph equipped with a 300 grooves $/ \mathrm{mm}$ grating, which provides an effective spectral window of $280 \mathrm{~nm}$ at the exit port of the spectrograph. The emission spectrum is recorded on an optical multichannel analyser based on an intensified 
photodiode array detector (OMA III system, EG\&G PARC Model 1406). A computer interface system is finally used for the storage and analysis of the recorded spectra.

Ablation thresholds have been defined as the $\mathrm{F}_{\text {LASER }}$ where visible surface modification on the film is observed under an optical microscope. It has to be noted that this is not necessarily the etching threshold often used in the literature.

\section{RESULTS AND DISCUSSION}

The presentation considers first the comparison of the nature of the photoproducts observed at the various wavelengths and subsequently the different fluence dependences of photoproduct amount remaining in the substrate following irradiation at the different wavelengths. Finally, the effect of irradiating with successive laser pulses is assessed. As described above, in the present work, the employed dopants include naphthalene and phenanthrene and their iodo-derivatives (NapI and PhenI), while the employed polymer substrates include poly(methyl methacrylate) (PMMA) and polystyrene (PS), which exemplify different chemical compositions and reactivity of their functional units. Results on the initial examination of neat PS polymer films and how their photochemical modifications compare with those of simple aromatic dopants are finally presented.

\subsection{Wavelength Dependence}

Wavelength is generally considered to be the most important parameter that needs specification in implementations of UV ablation [1-11]. Generally, irradiation at relatively strongly absorbed wavelengths is selected for ensuring efficient etching and good surface morphology. These are, in fact, usually the only criteria employed for selection of the optimal wavelength. In addition, the operation of a photochemical mechanism is sometimes invoked for irradiation at wavelengths absorbed strongly by photodissociable units. The operation of such a mechanism is considered to result in further decrease of the thermal "load" to the substrate and thus to minimization of thermal degradation.

As described in more detail elsewhere [19], we have not found thus far any evidence for different types of photoproducts in the irradiation of the 
NapX/PMMA ( $X=\mathrm{Br}, \mathrm{I})$, PhenI/PMMA and PhenI/PS systems at the three examined wavelengths $(193 \mathrm{~nm}, 248 \mathrm{~nm}$ and $308 \mathrm{~nm})$. In the case of PMMA and PS films doped with NapI $(<1 \%$ wt), the probe spectra recorded after ablation are in all cases dominated by the NapH-type emission at 320-340 nm. In the case of the PhenI dopant, the photoproduct spectra exhibit similarly the characteristic pattern of PhenH-type emission, although in this case, the spectral displacement and broadening from the emission band recorded for PhenH-doped polymers indicate that the major product(s) is(are) substituted derivative(s) of the basic phenanthrene skeleton. (Fig. 1). The formation of these NapH and PhenH-like products can be ascribed to the aryl (naphthyl-Nap, and phenanhthrenyl-Phen) radicals that are formed by the homolytic photodissociation of the precursors [20] reacting with nearby PMMA units (either by abstraction or by addition reactions). The important point for the present purposes is that no particular difference in the type of photoproducts is detected following irradiation of the weakly doped systems at the various wavelengths.

$$
\begin{aligned}
& \mathrm{ArX} \stackrel{\mathrm{h} v}{\longrightarrow} \mathrm{Ar}+\mathrm{X} \\
& \mathrm{Ar}+\mathrm{PMMA} \longrightarrow \text { ArH-like }
\end{aligned}
$$

Similarly, no particular difference is observed in the irradiation of samples with a higher NapI dopant concentration ( $\geq 1 \% \mathrm{wt}$ ), for which, the additional formation of $\mathrm{Nap}_{2}$ and fused aromatic compounds (perylene) is observed (Fig. 2) [18]. (In the case of the PhenI dopant, there is some evidence for formation of the corresponding recombination/fused products, but the spectral examination is not as conclusive as in the case of the NapX dopants). $\mathrm{Nap}_{2}$-type product, evidenced by the broad emission centered at $\approx 375 \mathrm{~nm}$, is observed at all examined wavelengths $(193 \mathrm{~nm}, 248 \mathrm{~nm}$ and $308 \mathrm{~nm}$ ) and so is the perylene product (emitting at $\sim 445 \mathrm{~nm}$ and $475 \mathrm{~nm}$ ) [21]. However, the depicted spectra do show a tendency for $\mathrm{Nap}_{2}$ and fused aromatic species formation with increasing wavelength (corresponding to a decreasing absorption coefficient of the substrate). Thus, it seems that the same type of photoproducts are formed at the three wavelengths examined here, but the relative efficiency of formation of the various photoproducts does change. However, at each specific wavelength the $\mathrm{NapH} / \mathrm{Nap}_{2}$ ratio changes also with fluence (decreasing with increasing fluence) so that the significance of the present observations can be assessed only after the cor- 
(a)

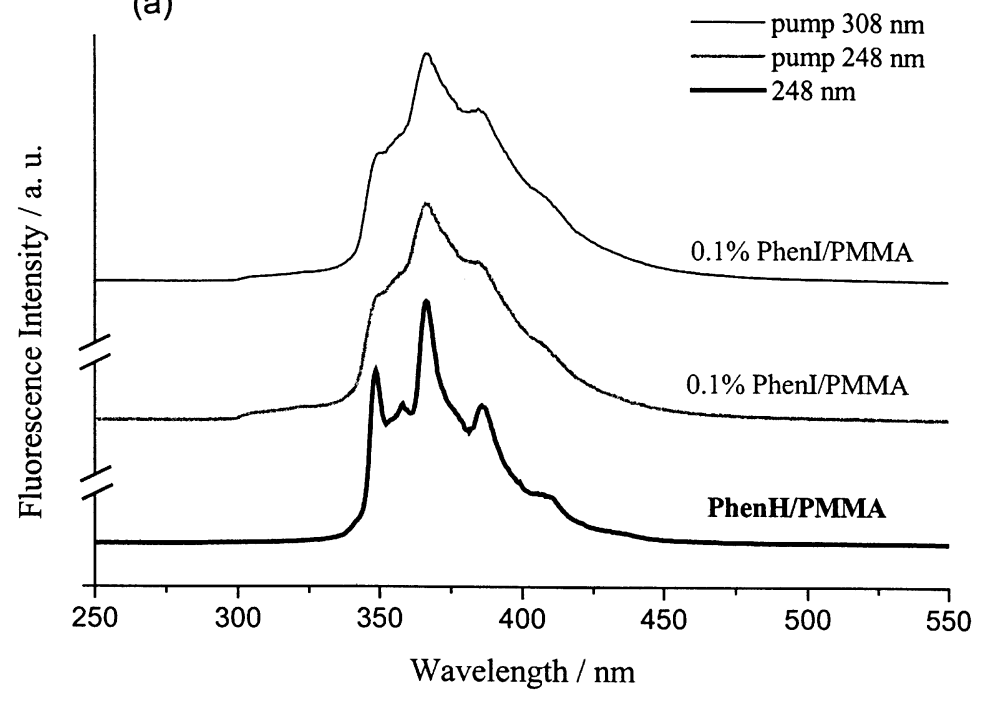

(b)

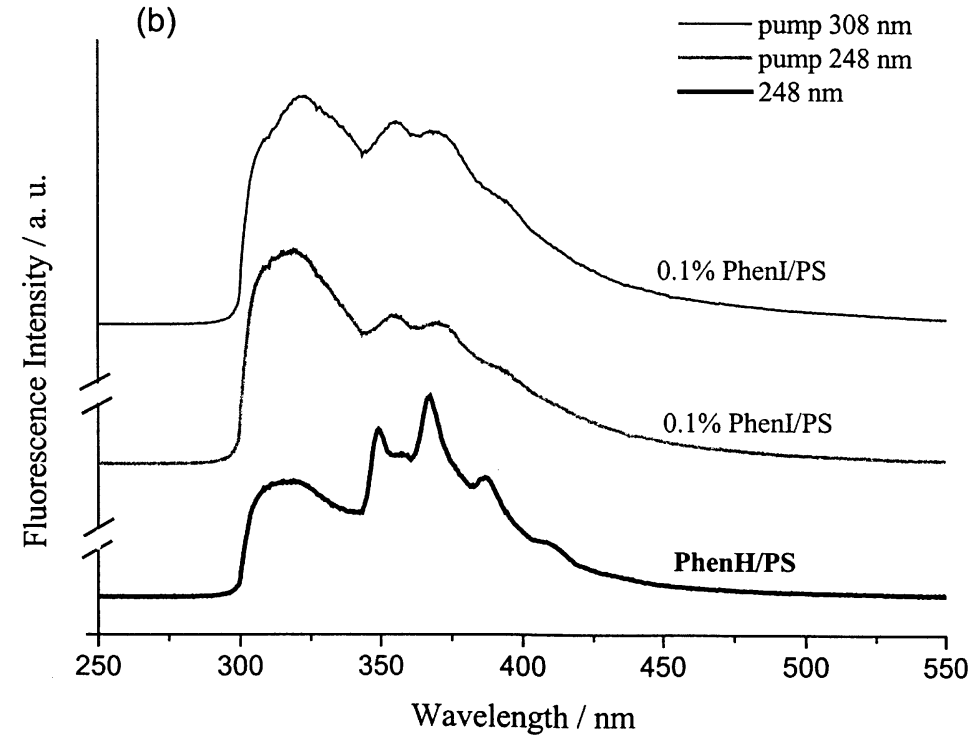

FIGURE 1 Laser-induced fluorescence spectra recorded from PMMA and PS samples doped with PhenI after their irradiation with a single "pump" pulse at the indicated wavelengths at laser fluences above the corresponding ablation thresholds. In the spectra from the PS samples, the broad emission at $\approx 310 \mathrm{~nm}$ is due to the polymer. For comparison purposes, the spectra have been arbitrarily scaled and spectra recorded from PhenH-doped PMMA and PS (0.1\% wt) are also included in the figure. 


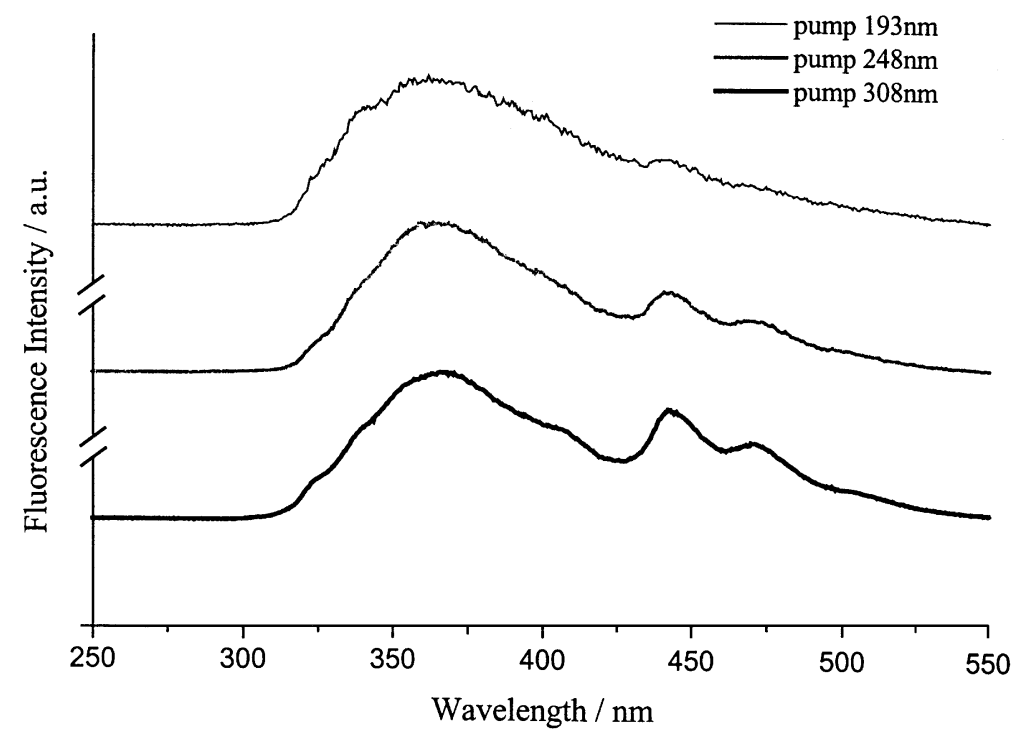

FIGURE 2 Probe fluorescence spectra recorded from NapI-doped PMMA samples of high dopant concentration (4\% wt NapI), after irradiation with a single "pump" pulse at the indicated wavelengths and laser fluences above the corresponding ablation thresholds.

responding thorough fluence examination. Furthermore, it should be noted that the previous remarks and conclusions refer only to the emitting photoproducts of the dopants, since these are the ones detected by the employed LIF technique. For a full mechanistic understanding of the importance of the wavelength in UV laser ablation of polymers, the non-fluorescing (dark) photoproducts must be also specified, even if these may be much less efficiently formed than the emitting ones. To this end, GC/MS examination has been initiated in parallel to the present fluorescence study.

\subsection{Dependence on Laser Fluence}

Much more informative for the importance of the wavelength turns out to be the quantitative characterization of the photoinduced effects as a function of laser fluence. To this end, the fluence dependence of the emission intensity (i.e., of the $\mathrm{NapH}$ precursor for $\mathrm{NapH} /$ polymer films or of the $\mathrm{NapH}$-like photoproduct for NapI/polymer films) following a single pump pulse on virgin polymer has been monitored. This intensity reflects 
essentially the amount of the fluorescing naphthyl species that remains in the substrate after irradiation.

Interestingly, even in the case of $\mathrm{NapH}$ dopant, quantitative changes are observed, namely a decrease in the precursor fluorescence with successive laser pulses and/or increasing laser fluence. The decrease in the NapH fluorescence may be ascribed to thermal desorption of the dopant, as indicated by Fukumura et al. [22] and/or to photooxidation/degradation. The spectral examination does not provide any evidence for the photopro$\operatorname{duct}(\mathrm{s})$, however, this may be due to its (their) very low or minimal fluorescence yield. The possibility of photodegradation is nevertheless indicated by a parallel strong decrease in the transmission of the film, which is consistent with the formation of photoproducts that absorb stronger than the $\mathrm{NapH}$ precursor. Indeed, NapH and related compounds are known to generate ${ }^{1} \mathrm{O}_{2}\left(\Delta_{g}\right)$ efficiently following photoexcitation [23]. This has a long lifetime within polymers $[24,25]$ and it can attack the aromatic to form the corresponding endoperoxide $\left(\mathrm{Nap}-\mathrm{O}_{2}\right)$. Such processes have been shown to occur in related polymer systems [23].

At present, the quantitative examination of this $\mathrm{NapH}$ phtotodegradation process has been examined in detail only in the case of irradiation at $248 \mathrm{~nm}$, though similar dependence is also indicated at the other wavelengths (Fig. 3). At low fluences, the decrease in the fluorescence of the $\mathrm{NapH}$ dopant, i.e. the extent of its photodegradation, is found to depend on $\mathrm{F}_{\text {LASER }}$. The dependence scales as $\mathrm{F}_{\text {LASER }}{ }^{1 / 2}$, which is consistent with the previously suggested intermediate formation of ${ }^{1} \mathrm{O}_{2}\left(\Delta_{g}\right)$. With increasing fluence, the process seems to saturate, reaching a limiting value near the ablation threshold (Fig. 3). Thus, UV ablation appears to result in a limitation of the extent of photodegradation that the NapH dopant undergoes.

Turning next to the consideration of the photodissociable NapI and PhenI dopants, the $\mathrm{F}_{\text {LASER }}$-dependence of the NapH-like photoproduct intensity (monitored at $\lambda=335 \mathrm{~nm}$ ) remaining in the substrate following irradiation with a single "pump" pulse is depicted in Figure 4. For the $0.4 \%$ wt samples, at $248 \mathrm{~nm}$ and $308 \mathrm{~nm}$, the linear increase of the photoproduct yield at low laser fluences is followed by a sharp rise above the threshold. Thus, as compared with irradiation at lower fluences, there is an enhancement in the amount of photoproducts that remain in the substrate following ablation. In contrast, in the irradiation at $193 \mathrm{~nm}$, the yield is found to reach a limiting value near the threshold (Fig. 4(b)). Furthermore, the maximum photoproduct intensity is much lower than that in the case of the irradiation at the 

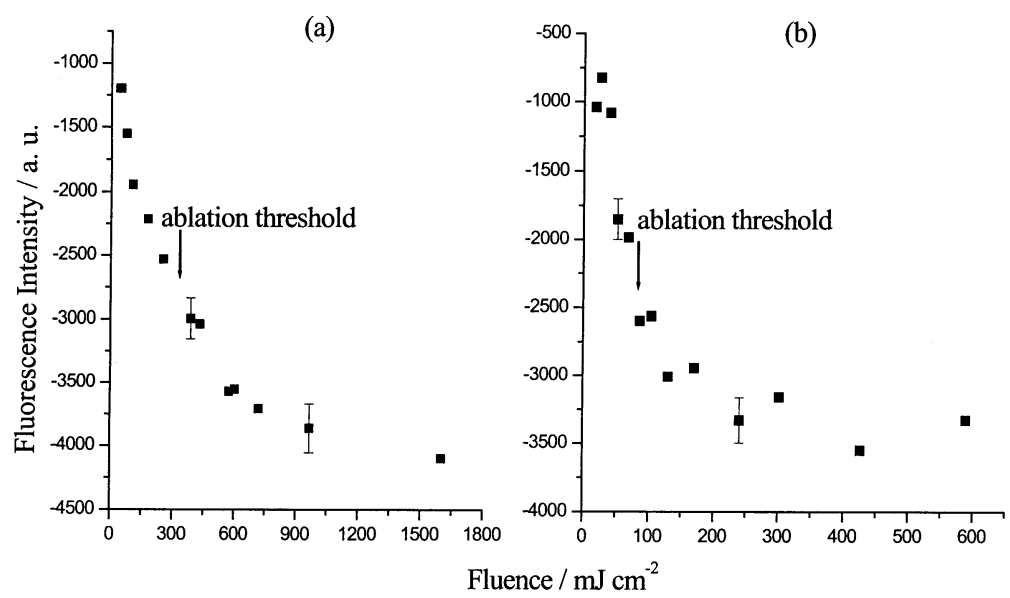

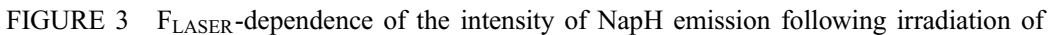
NapH-doped PMMA (0.4\% wt) with a single "pump" pulse at $248 \mathrm{~nm}$ (a) and at $193 \mathrm{~nm}$ (b). The error bars represent $2 \sigma$, as determined from at least 5 different measurements. The intensity decreases in the two graphs are not comparable, because they have been recorded under somewhat different excitation conditions.

$308 \mathrm{~nm}$ or $248 \mathrm{~nm}$ wavelengths. In other words, the amount of dopantderived photoproducts that remains in the substrate following ablation at $193 \mathrm{~nm}$ is much reduced from that in the irradiation at the other two wavelengths.

Since the spectral examination does not indicate any major qualitative differences between the three wavelengths, the difference in the $F_{\text {LASER }}$-dependence must relate mainly to the much higher absorptivity of PMMA at $193 \mathrm{~nm}\left(\varepsilon \approx 5 \times 10^{3} \mathrm{~cm}^{-1}\right)$ vs. that at the other two wavelengths $(248$, $308 \mathrm{~nm})\left(\varepsilon<500 \mathrm{~cm}^{-1}\right)$ [26-28]. To demonstrate this, similar experiments with polystyrene doped with iodo-phenanthrene (so that dopant emission is spectrally resolved from the strong polymer emission) were undertaken. In this case, a dependence similar to that observed for NapI/PMMA at $193 \mathrm{~nm}$ is observed for the PhenH-type photoproduct (monitored at $\lambda \approx 366 \mathrm{~nm}$ ) in the irradiation at $193 \mathrm{~nm}$ and $248 \mathrm{~nm}$, whereas the dependence at $308 \mathrm{~nm}$ is described by curve Figure 4(a). PS absorbs relatively strongly at both $193 \mathrm{~nm}$ and $248 \mathrm{~nm}$, but weakly at $308 \mathrm{~nm}$ [26, 29, 30]. Thus, for both polymers, there appears to be a close correlation between the absorption

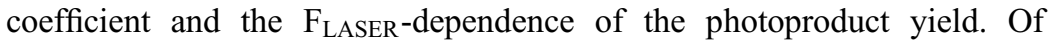
course, this conclusion does not imply that the chemical composition of 

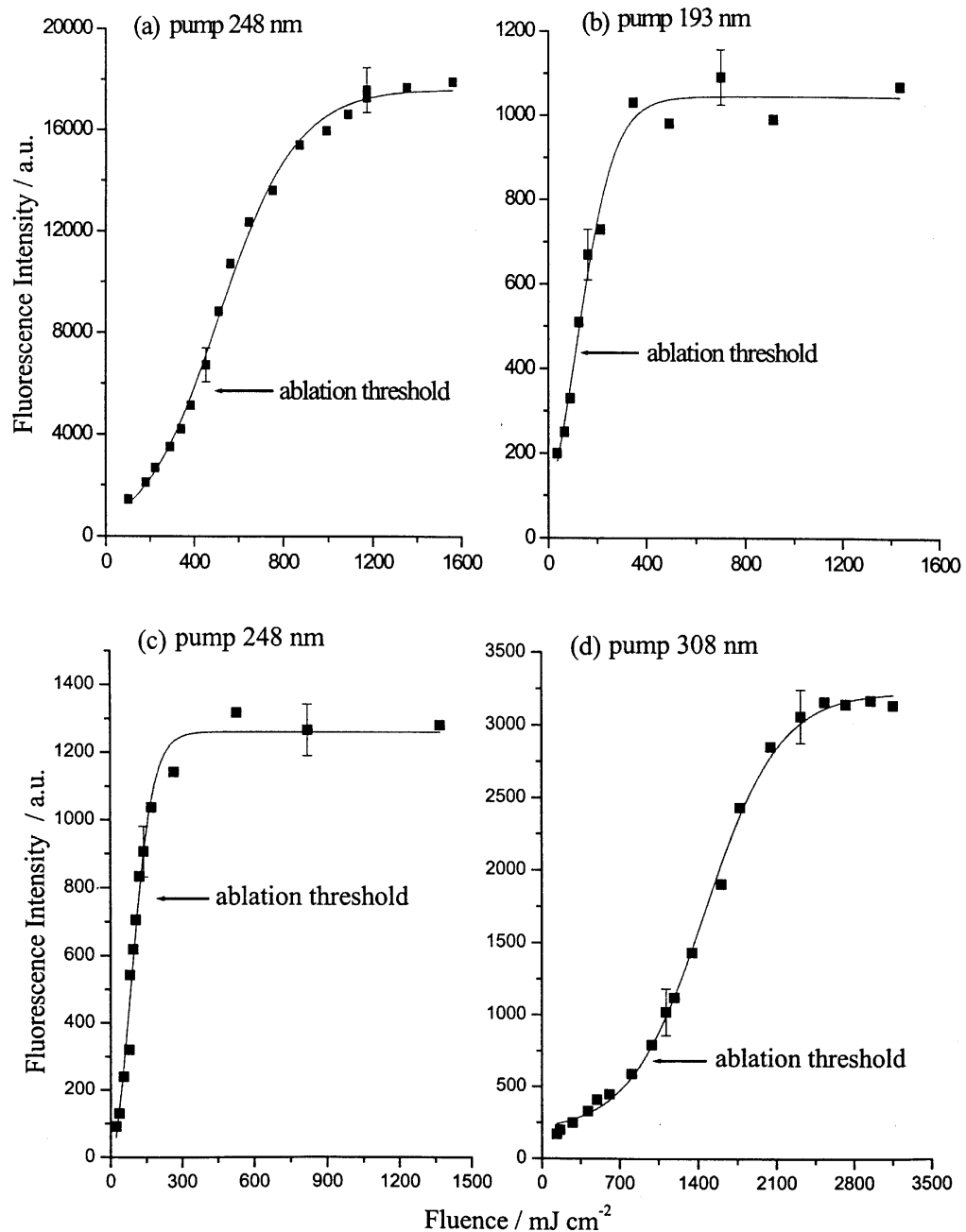

FIGURE $4 \quad \mathrm{~F}_{\text {LASER }}$-dependence of the NapH-like (monitored at $\lambda=335 \mathrm{~nm}$ ) and PhenH-like (monitored at $\lambda=366 \mathrm{~nm}$ ) photoproduct emission in the irradiation of $0.4 \% \mathrm{wt} \mathrm{NapI} / \mathrm{PMMA}$ (top figures) and $0.1 \%$ wt PhenI/PS (bottom figures) at the indicated wavelengths. The error bars represent $2 \sigma$, as determined from at least 5 different measurements. 
the matrix is not significant, but that, at least to a first order approximation and for the systems studied here, it is of secondary importance to the absorptivity.

At present, two possibilities can account for this importance of the absorption coefficient. The first possibility concerns a much more effective light absorption by the ejected plume at stronger absorbed wavelengths (i.e., for $0.4 \% \mathrm{wt} \mathrm{NapI/PMMA,} \mathrm{at} 193 \mathrm{~nm}$ than at 248 and for PhenI/PS, at $248 \mathrm{~nm}$ and $193 \mathrm{~nm} v s$. at $308 \mathrm{~nm}$ ). Indeed, the importance of plume absorption in determining the extent of etching depth has been considered extensively in the literature [31-33]. Alternatively, this dependence can be understood by the fact that the absorption coefficient determines the relative ratio of etching depth vs. optical penetration depth and thus the depth over which photoproducts that remain in the substrate with increasing laser fluence. However, the quantitative analysis of the results requires an accurate knowledge of the effective absorption coefficient, which generally differs significantly during ablation from the linear one. To this end, temporally-resolved absorption measurements are currently in progress. In parallel, plasma emission studies are under way for establishing the relative importance of the two plausible explanations.

Independently of the mechanisms responsible for the observed dependence, this result clearly indicates that a key factor for the success of the various implementations of UV ablation relates to the high absorptivity of the processed molecular substrates. Indeed, in most applications, best results are attained via the use of irradiation wavelength that is strongly absorbed by the treated substrate. It is generally assumed that the necessity for irradiation at strongly absorbed wavelengths is due to the need for efficient etching, and good morphology of the treated area. However, according to the present work, the strong absorption also indicates that the induced photochemical modifications may be closer to the ones indicated in Figure 4(b) and 4(d), i.e., highly reduced from that expected on the basis of the linear photochemistry that occurs at low laser fluences. The importance of the wavelength is further enhanced by the indication of Figure 2 for a reduction in the formation of recombination/condensation products with increasing absorptivity. Thus, the present work demonstrates that besides the improved etching efficiency and substrate morphology, an additional reason for the importance of strongly absorbed wavelength relates to the high degree of "photochemical protection" afforded to the remaining material. 
The importance of high absorptivity of the processed molecular substrates has, for example, been demonstrated in the case in the laser-restoration of painted artworks. In this application, irradiation with excimer laser pulses is employed to effect removal of the outer degraded layers of varnish. Varnish is a mixture of strongly UV-absorbing organic substances and is applied on top of the paintings for aesthetic and protective purposes [7]. However, with time, it undergoes photooxidation at its outer layers, with detrimental effects in the appearance and even for the integrity of the painting, thereby necessitating its removal. Indeed, irradiation with excimer lasers has been shown to enable a highly efficient removal of these degraded layers. Interestingly, it has been found empirically that if the etching is stopped before complete removal of the varnish covering the artwork, minimal if any alterations in the underlying medium are detected. In contrast, if irradiation is continued until the complete removal of varnish, then extensive modifications and degradation of the pigments is observed. These results can be easily rationalized within the framework of the previous work on the doped polymer films. The high absorptivity $\left(\alpha \approx 10^{5} \mathrm{~cm}^{-1}\right)$ of varnish ensures, on one hand, that excimer laser irradiation effects efficient etching and material removal, and on the other, that photochemical effects are localized within $\mathrm{d}<1-5 \mu \mathrm{m}$, at most, from the surface [8].

\subsection{Dependence on Number of Successive Laser Pulses}

Another parameter that is usually important in practical implementations is the number of pulses required for the removal of the undesirable surface layers. Thus, the dependence of the extent of photochemical modifications on the number of pulses employed to irradiate the substrate at a given area is of direct relevance for applications.

Figure 5 depicts the fluorescence intensity at $\lambda=335 \mathrm{~nm}$ (corresponding to the NapH-like photoproduct) as a function of successive "pump" laser pulses (that have irradiated the same spot) for irradiation of $0.4 \% \mathrm{wt}$ $\mathrm{NapI} / \mathrm{PMMA}$ at various fluences at $248 \mathrm{~nm}$. Below the threshold, there is an accumulation of the NapH-like photoproduct in the film, described expectably by an exponential of the form $\left[1-\exp \left(-\alpha \mathrm{F}_{\text {LASER }} \mathrm{N}\right)\right]$, where $\mathrm{N}$ is the number of pulses, until finally a plateau is reached. In contrast above the threshold, photoproduct fluorescence is observed to decrease after the first few pulses. The decrease can be ascribed to material removal by the etching 
(a)

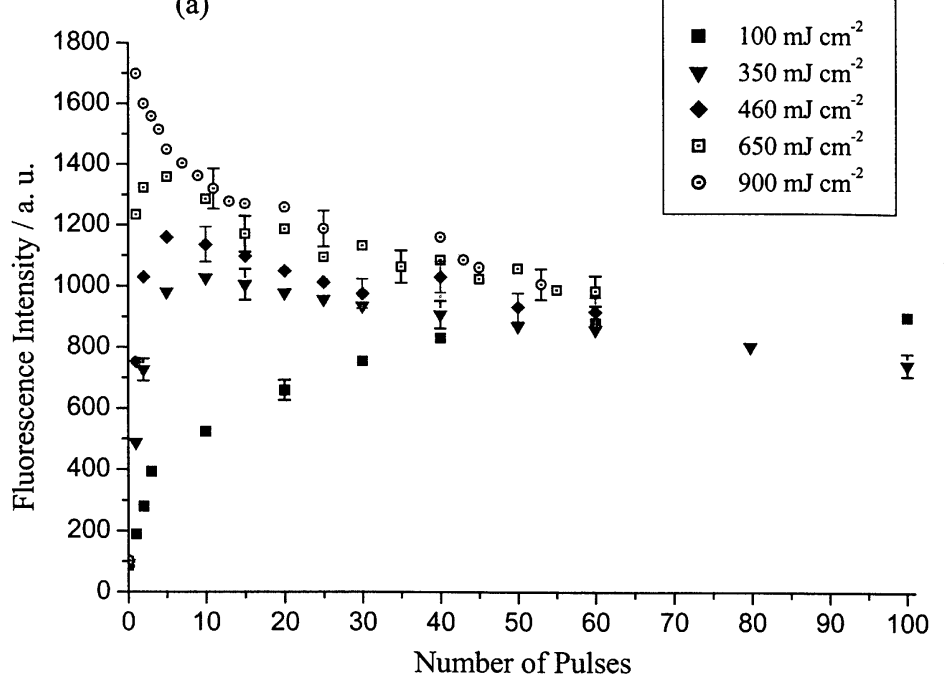

(b)

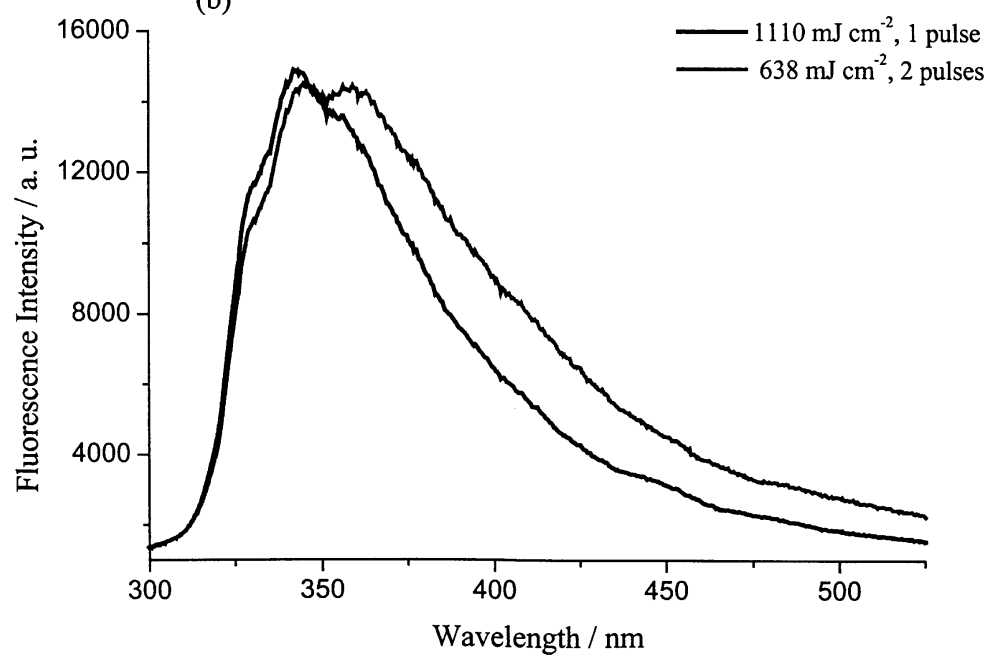

FIGURE 5 (a) The intensity of NapH-like photoproduct emission product emission $(\lambda=335 \mathrm{~nm})$ as a function of the number of successive pump laser pulses on NapI-doped $(0.4 \% \mathrm{wt})$ PMMA, at indicative laser fluences below, near and above the ablation threshold. The error bars represent $2 \sigma$, as determined from at least 5 different measurements. (b) Laserinduced fluorescence spectra recorded from NapI/PMMA $(0.4 \% \mathrm{wt})$ irradiated with the indicated number of pulses (at $248 \mathrm{~nm}$ ) at two fluences above the ablation threshold. 
process and also plausibly to the photooxidation/degradation of the NapHlike photoproduct discussed before.

A further feature concerning the importance of number of pulses derives from the spectral examination of the probe spectra from samples that have been irradiated at various fluences but approximately at the same total photon dose (Fig. 5). The comparison is meant to be qualitative, as the quantitative one requires correction for self-absorption and scattering effects by the laser modified film surface. Aside from the fact that no welldefined procedure for correcting for these effects is established in the literature, correction in the present case for changes with number of successive laser pulses is far from straightforward. Nevertheless, even the qualitative examination indicates an interesting feature: at all fluences, the spectra following the few initial pulses are dominated by the NapH-type photoproduct emission, but with continuing irradiation, they get progressively broader and red-shifted. Consequently, for irradiation at the same total photon dose but at different fluences, accumulation of by-products is higher with increasing higher number of pulses that have irradiated a given spot (i.e., higher for irradiation at lower fluences).

In all, the examination demonstrates that multipulse irradiation protocols must be avoided in the applications, since they result in higher and higher accumulation of ill-defined products and secondary reactivity. Of course, in the various implementations of UV ablation, the necessary number of pulses is specified by the etching efficiency as compared to the amount of material required to be removed. Thus, in practice, a compromise may have to be drawn between efficient material removal and minimization of photochemical effects.

\subsection{Examination of Neat PS Modifications}

As an extension of the study to more complex systems, we present also preliminary results on the oblation of PS films [34, 35]. The question addressed here concerns how the previous results differ for chromophores being part of the polymer chain rather than a "small" dopant dispersed within the polymeric matrix. To this end, we examine here PS, which is relatively strong fluorescing polymer.

The probe spectrum of PS consists essentially by an emission band at $320 \mathrm{~nm}$, in good agreement with the literature reports. [36-38] This emission is generally ascribed to excimer emission of the phenyl side groups 

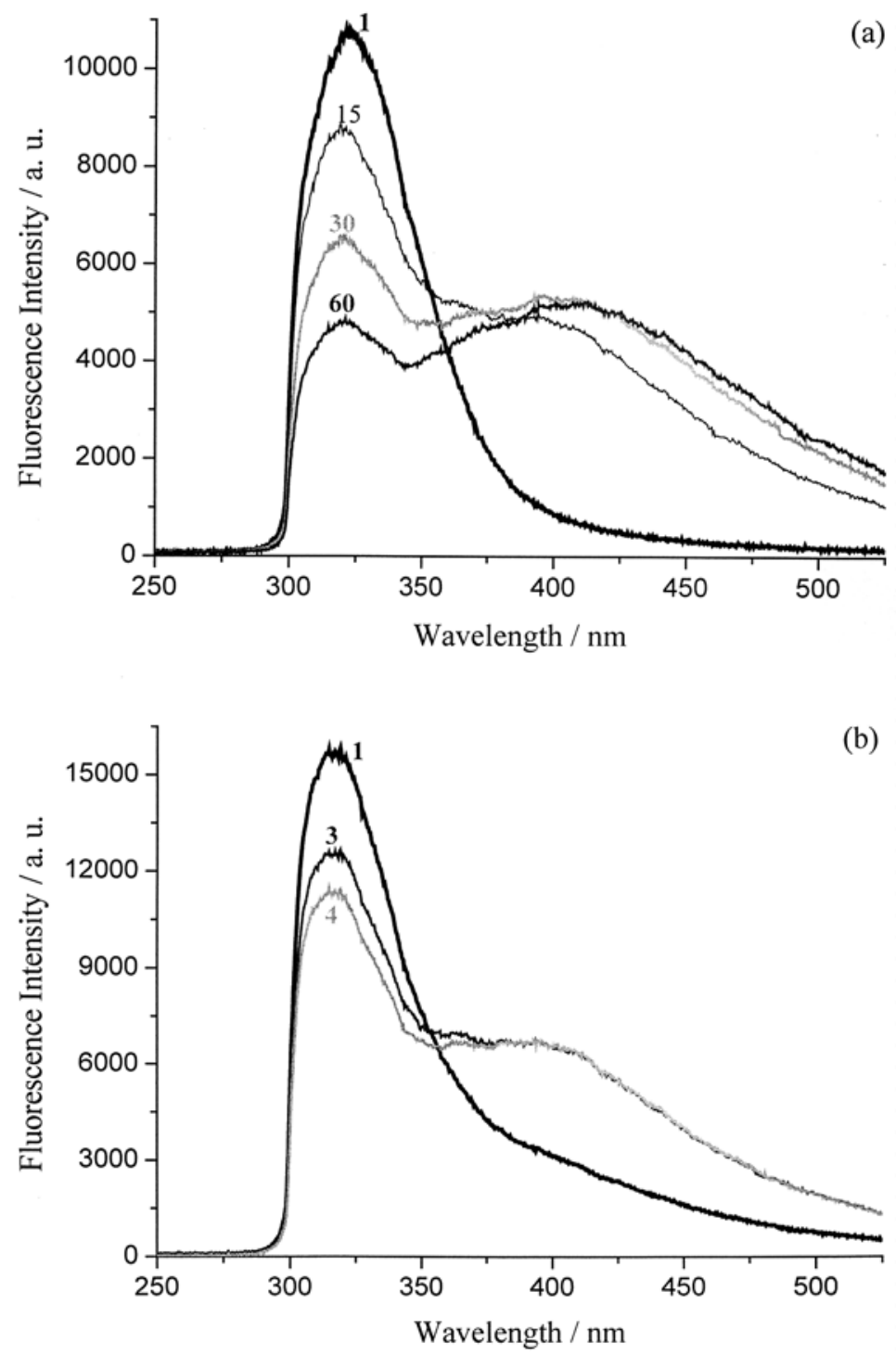

FIGURE 6 Laser-induced fluorescence spectra recorded from a PS film irradiated with the indicated number of pulses (at $248 \mathrm{~nm}$ ) at a fluence (a) below $\left(\mathrm{F}_{\text {LASER }} \approx 85 \mathrm{~mJ} / \mathrm{cm}^{2}\right)$ and (b) above the ablation threshold $\left(\mathrm{F}_{\text {LASER }} \approx 565 \mathrm{~mJ} / \mathrm{cm}^{2}\right)$. 
(monomer emission being mainly observed at $\approx 285 \mathrm{~nm}$ ). Figure 6 illustrates the spectra recorded from PS upon irradiation with successive laser pulses at low laser fluences. In this case, the fluorescence peak of PS is observed to decrease gradually to very low values, with the decrease per pulse being more pronounced with increasing laser fluence. In parallel, a broad emission band at $\approx 440 \mathrm{~nm}$, evidently due to highly conjugated products deriving from the polystyrene chains, is observed to grow with successive laser pulses. Based on previous studies, it is suggested that PS undergoes fragmentation to give benzyl and/or phenyl radicals, thereby accounting for the observed decrease in the excimer emission at $\approx 320 \mathrm{~nm}$, while the emission at $440 \mathrm{~nm}$ can be ascribed to polyene structures.

The important point for the present purposes concerns the pulse evolution observed in the irradiation above the threshold $\left(\approx 100 \mathrm{~mJ} \mathrm{~cm}^{-2}\right)$. Here, intense photoproduct emission is detected following the very first pulse, along with the decrease in the PS fluorescence. However, subsequent pulses do not appear to change much the ratio between the PS emission and that of photoproducts. Essentially, in this case, the ratio of degradation/PS emissions never turns to the extreme one observed at low laser fluences. There seems to be a balance between the degree of polymer degradation and the extent of photoproduct accumulation in the remaining substrate. Thus, the results seem to be largely analogous to the conclusion reached in the pulse evolution of the NapI photoproduct for irradiation at strongly absorbed wavelengths.

\section{CONCLUSIONS}

In summary, the dependence of the nature and of the extent of the modifications of dopants within PMMA and PS polymer films has been examined as a function of laser parameters (laser fluence, wavelength, and number of pulses) and chromophore/polymer properties. It is shown that the substrate absorptivity and the number of laser pulses employed turn out to be the critical parameters in determining the quantity of photoproducts remaining in the substrate. No particular dependence of the nature, at least of the probed emitting products formed at $308 \mathrm{~nm}, 248 \mathrm{~nm}$ and $193 \mathrm{~nm}$, on wavelength is observed, though there is an indication that the relative efficiency for formation of recombination and fused polyaromatic products is enhanced with decreasing substrate absorptivity. Most importantly, different 
types of $F_{\text {LASER }}$-dependence of the photoproduct amount remaining in the substrate are found for the different wavelengths. In the case of irradiation at strongly absorbed wavelengths, the amount of photoproduct remaining in the substrate following ablation is reduced from that expected on the basis of linear photochemistry, whereas in the irradiation at weakly absorbed wavelengths, the amount is indicated to be higher. Thus, it appears that absorptivity of the substrate at the irradiation wavelength is the major determinant for the type of dependence observed. The implications of these results for the optimisation of laser processing of molecular/organic solids are discussed and exemplified for the particular case of laser restoration of painted artworks as a study case. It is suggested that the importance of employing relatively strongly absorbed wavelengths in laser processing may relate, besides the efficient etching and good surface morphology, to the minimization of the chemical modifications. In contrast, irradiation with successive laser pulses is demonstrated to be disadvantageous for the photochemical integrity of the substrate, since it results in further photolysis and accumulation of photoproducts, as well as in secondary, ill-defined photoproducts.

In all, the study of these model systems appears to be most appropriate for establishing criteria for the systematic optimisation of laser processing techniques of molecular substrates. Furthermore, though the present study has focused on the implications for the laser processing of polymeric substrates, the results provide also information about the condition of the film and the nature of chemical processes in the UV ablation. These will be addressed elsewhere, but nevertheless the results clearly show that photochemistry (photofragmentation and subsequent reactivity) in UV ablation is non-trivially modified from that observed at low laser fluences.

\section{Acknowledgements}

The work was supported in part by the Ultraviolet Laser Facility operating at F.O.R.T.H. under the Improving Human Potential (IHP)-Access to Research Infrastructures programme (contract No. HPRI-CT-1999-00074) and by the Training and Mobility of Researchers (TMR) programme of the EU (project No. ERBCHRXCT930157) and by the חENE $\Delta$ programme (project No. 99E $\Delta 6$ ) of the General Secretariat of Reseach and Technology-Ministry of Development (Greece). A fellowship to E.A. by the EПЕAEK programme "Applied Moleculary Spectroscopy" 
(Department of Chemistry, University of Crete) is gratefully acknowledged. The authors would like to thank Prof. G. Fytas and Dr. D. Vlassopoulos of the Polymer group at F.O.R.T.H. for helpful discussions on polymer properties.

\section{References}

[1] Boyd I. A. (Ed.), "Photochemical Processing of Electronic Materials", (Academic Press, London, 1992)

[2] (a) Miller J. C. (Ed.), "Laser Ablation. Principles and Applications", (Springer Ser. Mater. Sci. 28, Springer-Verlag, Berlin, 1994) (b) Baeuerle D., "Laser Processing and Chemistry, Springer, Berlin, 2000), Chap. 12-13 and 27

[3] for instance: (a) Rumsby P., Harvey E., Thomas D., Rizvi N., (1997) SPIE Vol. 3184, 176 and references therein (b) Archambault J. L., Reekie L., Russel R. S. J., (1993) Electronics Letters 29 (1).

[4] (a) Radhakrishnan G., Marquez N. and Muller H., (1992) MRS Proc. 236, 77 (b) Lu Y. F., Lee Y. P., Zhan M. S., (1998) J. Appl. Phys. 83, 1677

[5] (a) Blanchet G. B., (1993) Appl. Phys. Lett. 62, 479 (b) Blanchet G. B., Fincher Jr. C. R., (1994) Adv. Mater. 6, 881 and references therein

[6] Tolbert W. A., Lee I. -Y. S., Doxtrader M. M., Ellis E. W. and Dlott D. D., (1993) J. Imaging Sci. Technol. 37, 411

[7] Zafiropulos V., Fotakis C., in "Laser Cleaning in Conservation: an Introduction", Cooper M. (Ed.) (Butterworth Heinemann, Oxford, 1998)

[8] Georgiou S., Zafiropulos V., Anglos D., Balas C., Tornari V. and Fotakis C., (1998) Appl. Surf. Sci. 127-129, 738

[9] "Laser-Tissue Interaction IX", ed. by Jacques S. L., SPIE Proc. Series, vol. 3254 (SPIE, Washington, 1998) and references therein

[10] Hill P., "Multiphoton excitation eradicates eye cancers," 2000 OLE 71, 29-31

[11] Gabrielly C. B., Pacella E., Abdolrahimzadeh S., Regine F., Mollo R., (1999) Ophthalmic. Surg. Las. 30, 442 and references therein

[12] (a) Brunco D. P., Thompson M. O., Otis C. E., Goodwin P. M., (1992) J. Appl. Phys. 72, 4344 (b) Chen S., Lee I.-Y. S., Tolbert W. A., Wen X. and Dlott D. D., (1992) J. Phys. Chem. 96, 7178 (c) Lee I. -Y. S., Wen X., Tolbert W. A., Dlott D. D., Doxtader M. and Arnold D. R., (1992) J. Appl. Phys. 72, 2440

[13] Lippert T., Langford S. C., Wokaun A., Georgiou S., Dickinson J. T., (1999) J. Appl. Phys. 86, 7116

[14] Zweig A. D., Venugopalan V. and Deutch T. F., (1993) J. Appl. Phys. 74

[15] Siano S., Pini R. and Salimbeni R., (1999) Appl. Phys. Lett. 74, 1233

[16] (a) Srinivasan R., Braren B., Dreyfus R. W., Handel L. and Seeger D. E., (1986) J. Opt. Soc. Am. B3, 785 (b) Sutcliffe E., Srinivasan R., (1986) J. Appl. Phys. 60, 3315

[17] Webb R. L., Langford S. C., Dickinson J. T., Lippert T. K., (1998) Appl. Surf. Sci. 127129, 815

[18] Berlman I. B., "Handbook of Fluorescence Spectra of Aromatic Molecules" (Academic Press, New York, 1971).

[19] (a) Athanassiou A., Andreou E., Anglos D., Georgiou S. and Fotakis C., (1999) Appl. Phys. A 86, S285 (b) Lassithiotaki M., Athanassiou A., Anglos D., Georgiou S. and Fotakis C., (1999) Appl. Phys. A, Rapid Comm. 69, 363 (c) Athanassiou A., Andreou E., Fragouli D., Anglos D., Georgiou S., Fotakis C., Journal of Photochemistry \& Photobiology: Chemistry (to appear)

[20] Haselbach E., Rohner Y. and Suppan P., (1990) Helv. Chim. Acta 73, 1644

[21] Offen H. W. and Beardslee R. A., (1968) J. Chem. Phys. 48, 3584 
[22] Fukumura H., Mibuka N., Eura S., Masuhara H. and Nishi N., (1993) J. Phys. Chem. 97, 13761

[23] (a) Twarowski A. J., (1988) J. Phys. Chem. 92, 6580 (b) Twarowski A. J. and Dao P. J., (1988) J. Phys. Chem. 92, 5292

[24] Gao Y., Baca A. M., Wang B. and Ogilby P. R., (1994) Macromolecules 27, 7041

[25] Wang B. and Ogilby P. R., (1995) Can. J. Chem. 73, 1831

[26] Meyer J., Kutzner J., Feldmann D., Welge K. H., (1988) Appl. Phys. B45, 7

[27] Cain S. R., Burns F. C., Otis C. E., Braren B., (1992) J. Appl. Phys. 72, 5172

[28] Küper S., Stuke M., (1987) Appl. Phys. B44, 199

[29] Lazare S., Granier V., (1988) J. Appl. Phys. 63, 2110

[30] Bolle M., Lazare S., (1993) J. Appl. Phys. 73, 3516

[31] Beinhorn F., Ihlemann J., Luther K., Troe J., (1999) Appl. Phys. A 68, 709

[32] Lazare S and Granier V., (1989) Laser Chem. 10, 25

[33] Hopp B., Csete M. and Szabo G., (1995) Appl. Phys. A61, 339

[34] Tsuboi Y., Sakashita S. I., Hatanaka K., Fukumura H. and Masuhara H. (1996), Laser Chem. 16, 167.

[35] Tsunekawa M., Nishio S., and Sato H. (1995), Jpn. J. Appl. Phys. 34, 218

[36] Gupta M. C., Gupta A., Horowitz J. and Kliger D., (1982) Macromolecules 15, 1372

[37] Vala Jr., M. T., Haebig J. and Rice S. A., (1965) J. Chem. Phys. 43, 886

[38] Rabek J. F., "Mechanisms of Photophysical Processes and Photochemical Reactions in Polymers: Theory and Applications" (John Wiley: Chichester, 1987) 